\title{
Pré-Comp: introduzindo os fundamentos da Computação e contribuindo com a motivação e aproveitamento acadêmico
}

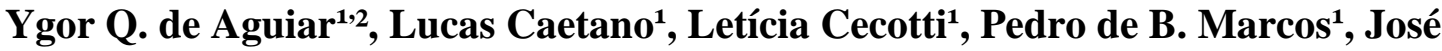 \\ R. Azambuja ${ }^{1}$ \\ ${ }^{1}$ Centro de Ciências Computacionais - C3 \\ 2Programa de Educação Tutorial Ciências Computacionais - PET-C3 \\ Universidade Federal do Rio Grande (FURG) - Rio Grande, RS - Brazil \\ \{ygorqa, lucas.caetano, leticiacecotti, pbmarcos, \\ jrazambuja\}@furg.br
}

\begin{abstract}
Computer science education, as well as the development of competencies and skills related to technology, features a large relevance for development of computational thinking and logical reasoning, contributing to the problem-solving ability. However, the number of enrollments in computing has undergone reduction and, for this reason, the performance of students in introductory courses proves to be a concern for educators in the area. This article presents the Pre-Comp, a project that besides contributing to the foundation of the basics of computing also helps with familiarization with academia and prospects in the professional field as well as for the development of creativity, interest and motivation of students.
\end{abstract}

Resumo. O ensino em computação, assim como o desenvolvimento de competências e habilidades ligadas à tecnologia, é um fator muito importante para o desenvolvimento do pensamento computacional e o raciocínio lógico, contribuindo com a capacidade de resolução de problemas. No entanto, o número de interessados em computação vem sofrendo redução e, por este motivo, o desempenho dos estudantes nas disciplinas introdutórias demonstra ser uma preocupação para os educadores na área. Esse artigo apresenta o Pré-Comp, um projeto que além de contribuir com a fundamentação dos princípios básicos da computação também contribui com a familiarização com o meio acadêmico e perspectivas no campo profissional, assim como para o desenvolvimento da criatividade, interesse e motivação dos estudantes.

\section{Introdução}

O ensino da computação e tecnologia de uma maneira geral tem empenhado um grande papel visto a importância do desenvolvimento e pesquisa nessas áreas na atualidade. Por este motivo, existe um crescente interesse em trabalhos que visam compreender como o processo de ensino em computação tem sido eficaz, inovador e ao mesmo tempo dinâmico ao ponto de acompanhar a rápida evolução da ciência e tecnologia. No entanto, pesquisas nos Estados Unidos apontaram que o interesse em estudar computação tem diminuído ao decorrer dos anos no país. Por exemplo, [Simon 2009] mostrou que o número de estudantes em computação caiu $50 \%$ comparando o ano de 


\section{CBIE-LACLO 2015}

Anais dos Workshops do IV Congresso Brasileiro de Informática na Educação (CBIE 2015)

2002 e 2007. E [Tew 2005] afirma que a taxa de reprovação em disciplinas básicas em computação se encontra em torno de $30 \%$.

Portanto, vários trabalhos buscam práticas pedagógicas inovadoras para atender a necessidade do ensino em computação e tecnologia [Tew 2005, Wick 2007, McWhorter 2009]. Considerando a realidade dos anos iniciais dos cursos em Engenharia de Computação, Engenharia de Automação e Sistemas de Informação do Centro de Ciências Computacionais (C3) da Universidade Federal do Rio Grande (FURG), o presente artigo descreve uma atividade pedagógica realizada com o intuito de possibilitar um maior aproveitamento no ensino dos fundamentos básicos de computação presentes na grade curricular dos cursos supracitados, assim como a integração e relação com as demais disciplinas do primeiro ano.

\section{Problemática e Objetivo}

Atualmente a maior parte dos alunos ingressos no ensino superior provem diretamente do ensino médio, caracterizando um público juvenil e muita das vezes imaturo para o meio acadêmico. O Programa de Apoio a Planos de Reestruturação e Expansão das Universidades Federais Brasileiras (REUNI) é uma iniciativa do Governo Federal para dar às instituições de ensino condições de expandir o acesso e garantir condições de permanência no Ensino Superior que foi instituído em 2007, no Plano de Desenvolvimento de Educação do MEC [Ministério da Educação 2007]. Após o surgimento do REUNI, os cursos universitários se expandiram consideravelmente, passando a oferecer um maior número de vagas numa maior quantidade de cursos. Esse programa favoreceu a criação de dois cursos que estão diretamente ligados com o escopo do projeto desenvolvido na nossa unidade acadêmica, o curso de Engenharia de Automação e Sistemas de Informação.

Outro fator que favoreceu o acesso ao ensino superior foi o Sistema de Seleção Unificada (SISU), desenvolvido pelo Ministério da Educação para selecionar os candidatos às vagas das instituições públicas de ensino superior que utilizam apenas a nota do Exame Nacional do Ensino Médio (ENEM) como fase única de seu processo seletivo. Após esses incentivos para expansão do acesso a educação superior é possível observar características proeminentes no perfil dos estudantes ingressantes. Ao aceitar uma maior quantidade de estudantes, a exigência de conhecimento requerido pelo SISU diminuiu e, consequentemente, também diminuiu a qualidade técnica dos estudantes. Outra característica marcante é a presença de estudantes de diversos estados do País, aumentando consideravelmente a diversidade em vários aspectos dentro das salas de aula [Andrade 2013, Silveira 2015].

Com o ingresso de alunos muita das vezes despreparados para o meio acadêmico, pois este se diferente muito em relação ao ensino médio e fundamental, o choque nas primeiras semanas de aula é evidente e inevitável. Somado com a deficiência encontrada no ensino de ciências exatas nas redes públicas de ensino, os alunos encontram dificuldades nas disciplinas da matemática e física e abdicam do tempo de estudo destinado às disciplinas da computação. Isso acarreta num aproveitamento muito inferior ao esperado, criando assim um alto percentual de reprovação nas disciplinas do primeiro ano, ou até mesmo comprometendo o bom 
aproveitamento das disciplinas subsequentes devido ao déficit nas disciplinas fundamentais do primeiro ano.

Portanto, identificando o perfil dos ingressantes, reconhecendo o déficit nos fundamentos de disciplinas básicas e a imaturidade acadêmica combinado com a desmotivação profissional é imprescindível a realização de alguma atividade pedagógica motivacional e de suporte que combate os altos índices de reprovação, retenção e principalmente evasão dos cursos do ensino superior. A atividade proposta se chama Pré-Comp e tem como público alvo os alunos do primeiro ano dos cursos de Engenharia de Automação, Engenharia de Computação e Sistema de Informação. O Pré-Comp, além de um curso preparatório para as disciplinas fundamentais de computação do primeiro ano, é composto por palestras e aulas motivacionais que instigam a curiosidade e o interesse dos alunos pelo desenvolvimento da ciência e tecnologia. Toda estratégia de ensino precisa estar diretamente envolvida com o âmbito motivacional dos estudantes. Isso porque um alto índice de motivação afeta positivamente na percepção dos estudantes em relação as disciplinas, o curso e a profissão almejada [Mitchell 2000, Stipek 1988]. Com isso, podemos elencar uma série de metas que desejamos alcançar com as atividades propostas:

- Apresentar a estrutura da Universidade não somente de maneira formal, mas também de maneira informal através de alunos veteranos e ex-alunos;

- Promover a integração dos estudantes dos três diferentes cursos envolvidos e desenvolver o perfil de trabalho colaborativo e em equipe;

- Introduzir os fundamentos de computação;

- Motivar os estudantes com relatos de alunos veteranos e egressos sobre a sua experiência na Universidade, no curso e sobre o mercado de trabalho e perspectivas do futuro na área.

\section{Metodologia}

O projeto Pré-Comp é composto por ciclo de palestras, aulas e exercícios práticos durante as semanas três e quatro do período letivo, no horário inverso às aulas. Durante a primeira semana letiva, o projeto é amplamente divulgado entre o calouros através do grupo PET Ciências Computacionais, e sua participação incentivada pelo corpo docente e direção do Centro de Ciências Computacionais (C3), unidade acadêmica responsável pela oferta dos cursos em questão. O Programa de Educação Tutorial (PET) é um programa do governo que compromete-se em contribuir com atividades extracurriculares na graduação que integrem o ensino, pesquisa e extensão, para a melhoria do ensino superior no país e proporcionar uma formação de excelência [Zimpeck 2012].

Na primeira fase, com duração de um dia, são realizados encontros de todos os calouros com a direção da unidade acadêmica e posteriormente com alunos veteranos e egressos. O principal objetivo desta fase é introduzir a Universidade e os cursos aos calouros formalmente (através da direção) e informalmente (através de relatos de alunos veteranos e egressos). 
Terminada a primeira etapa, os alunos são divididos em três grupos, de acordo com o curso de admissão na Universidade. Estes grupos são então individualmente apresentados à coordenação de cada curso através de encontros com os coordenadores e com alunos veteranos e egressos dos cursos. Desta maneira, objetiva-se apresentar individualmente cada um dos cursos de maneira formal (através das coordenações) e informal (através de relatos de alunos veteranos e egressos) aos calouros.

Numa terceira etapa, todos os calouros são divididos em grupos de até sete pessoas, sob a tutoria de alunos veteranos previamente selecionados dos cursos de graduação em questão. Cada grupo é individualmente treinado pelos seus tutores, ou como comumente chamados "treinadores", e é indispensável a diversidade dos grupos que contêm alunos dos três diferentes cursos. O principal objetivo das aulas é apresentar uma fundamentação da computação, principalmente trabalhar o pensamento computacional e o raciocínio lógico. Como ementa das aulas foi atribuído o conceito e tipos de representação de algoritmos (descrição narrativa, fluxograma e pseudocódigo), e conceitos como arranjo, vetor, matriz, ponteiro, sub-rotina e operadores (lógicos, aritméticos e relacionais). Nenhuma linguagem de programação é ensinada durante o Pré-Comp visto que o foco principal é construir o pensamento computacional e oferecer ferramentas para que posteriormente o algoritmo para solução de um problema possa ser facilmente traduzido para uma linguagem. Ao fim de cada treinamento é realizada uma prova prática a fim de avaliar cada grupo.

Durante as duas semanas de aulas e palestras, os grupos são avaliados pelos tutores e competem entre si pelos melhores resultados. Ao final da segunda semana, o grupo campeão recebeu um troféu entregue pelo diretor do C3 durante a Jornada de Integração do Centro de Ciências Computacionais (JIC3) promovido pelo grupo PET.

Ainda durante a terceira etapa, os alunos receberam palestras de egressos que se encontram no mercado de trabalho ou em programas de pós graduação, praticando os conhecimentos agregados durante a graduação e/ou dando continuidade aos estudos no campo.

\section{Resultados e Discussões}

$\mathrm{Na}$ elaboração do projeto pressupõe-se que os principais motivos de retenção e evasão são o pouco conhecimento sobre a estrutura e perspectivas profissionais dos cursos e acima de tudo a falta de motivação para superar as disciplinas básicas. Duas edições do Pré-Comp já foram realizados, no ano de 2014 e 2015. Nas próximas subseções somente os resultados obtidos na primeira edição são analisados para cada curso envolvido no projeto, visto que o ano letivo vigente ainda não encerrou.

\subsection{Engenharia de Computação}

A participação dos estudantes do curso de Engenharia de Computação na primeira edição do projeto foi bastante expressiva em relação aos outros dois cursos envolvidos. Isso pode ser facilmente observado quando comparamos os gráficos das taxas de aprovação dos três cursos. O Gráfico 1 apresenta a taxa de aprovação de três disciplinas introdutórias em computação do curso de Engenharia de Computação, em 3 anos consecutivos. Note que as disciplinas de Introdução à Ciência da Computação e 
CBIE-LACLO 2015

Anais dos Workshops do IV Congresso Brasileiro de Informática na Educação (CBIE 2015)

Matemática Discreta sofreram um aumento considerável. No ano de 2013, todas as disciplinas sofreram uma queda na taxa de aprovação comparando com o ano anterior. O Pré-Comp foi realizado no ano de 2014, com alta participação por parte dos estudantes deste curso e isso refletiu positivamente às taxas de aprovação das disciplinas no mesmo ano. A média total de aprovação nas disciplinas em pesquisa foi de $51 \% \mathrm{em}$ 2012; com uma queda significativa no ano seguinte, $37,2 \%$ em 2013, e no ano que foi realizado o projeto foi de $51,8 \%$, em 2014.

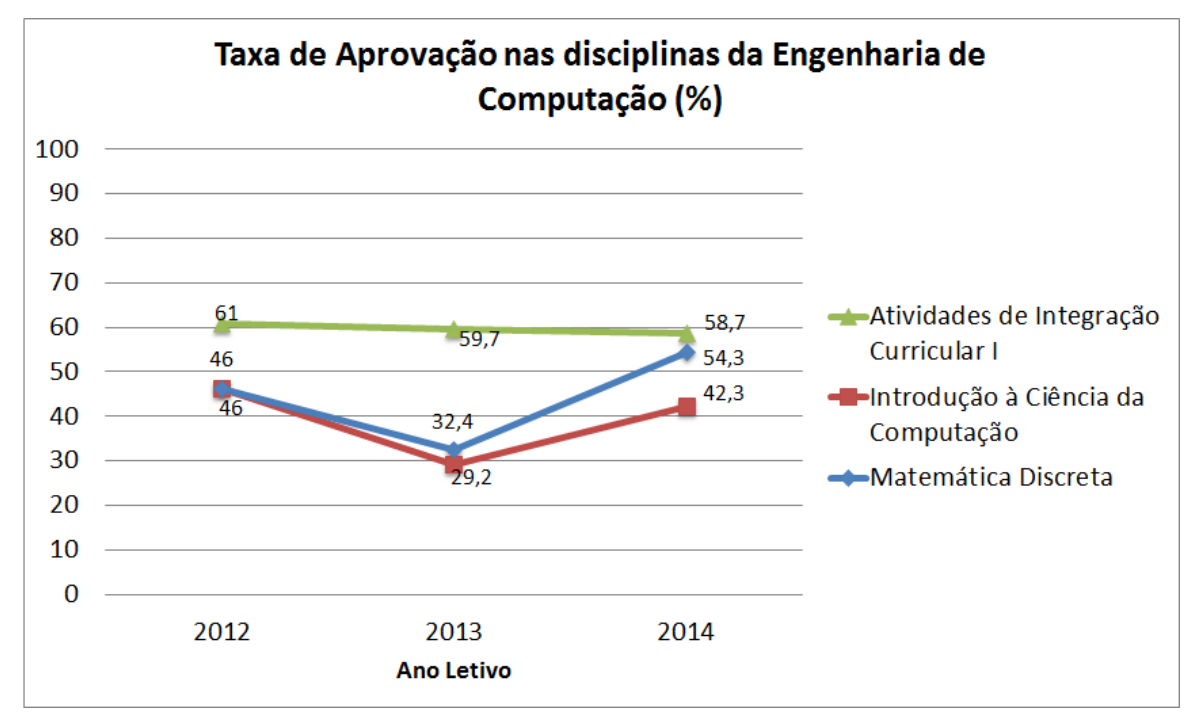

Gráfico 1. Taxa de Aprovação nas disciplinas de computação no primeiro ano do curso de Engenharia de Computação, em porcentagem (\%).

\subsection{Engenharia de Automação}

No curso de Engenharia de Automação, a participação no Pré-Comp não foi tão alta quanto no curso de Engenharia de Computação, talvez devido a falta de conhecimento que o curso em questão além de ter os fundamentos no campo da elétrica e mecânica também se fundamenta principalmente na computação. Esse comportamento foi observado através das dinâmicas das atividades onde os tutores puderam compreender melhor o perfil dos estudantes, confirmando o que foi previamente levantado em relação a desmotivação dos estudantes e imaturidade no meio acadêmico. No entanto, analisando o Gráfico 2 também se pode observar uma redução na taxa de aprovação nas disciplinas do ano de 2012 para 2013 e um aumento no ano da realização do projeto, em 2014, com uma média total de aprovação de 54,4\%. 
CBIE-LACLO 2015

Anais dos Workshops do IV Congresso Brasileiro de Informática na Educação (CBIE 2015)

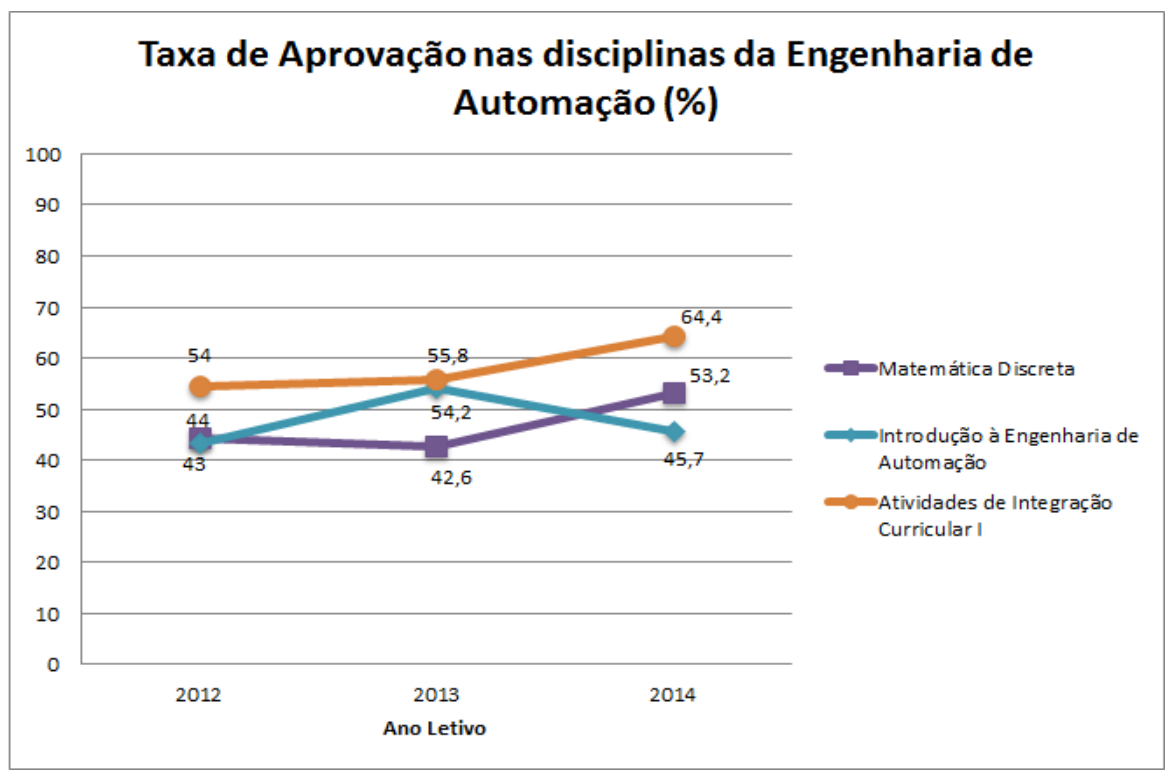

Gráfico 2. Taxa de Aprovação nas disciplinas de computação no primeiro ano do curso de Engenharia de Automação, em porcentagem (\%).

\subsection{Sistemas de Informação}

A participação dos estudantes do curso de Sistema de Informação foi surpreendentemente negativa podendo ser considerada nula. E esse comportamento refletiu diretamente na taxa de aprovação das disciplinas de computação nos anos iniciais do curso. No Gráfico 3 podemos observar a situação das disciplinas introdutórias em computação no curso de Sistemas de Informação no período dos últimos três anos. A média total de aprovação considerando as três disciplinas em pesquisa foi de apenas 30\% em 2014.

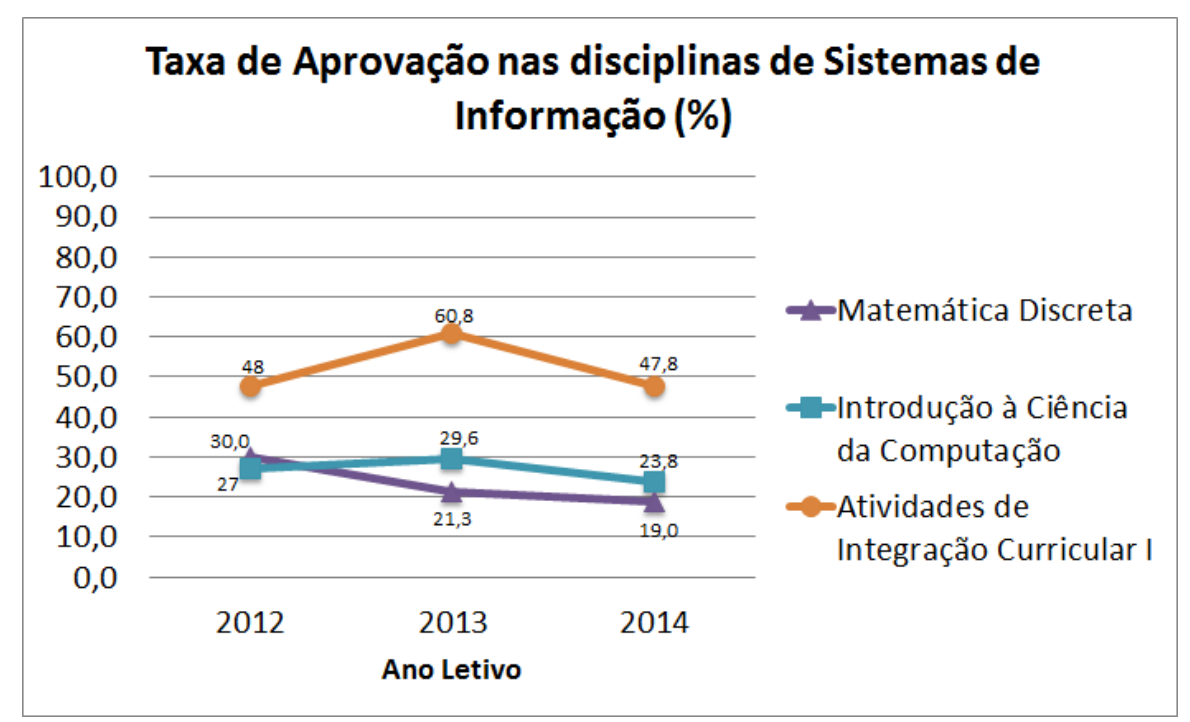

Gráfico 3. Taxa de Aprovação nas disciplinas de computação no primeiro ano do curso de Sistemas de Informação, em porcentagem (\%). 
CBIE-LACLO 2015

Anais dos Workshops do IV Congresso Brasileiro de Informática na Educação (CBIE 2015)

\section{Conclusão}

Este projeto planeja utilizar o período invertido às aulas dos cursos de Engenharia de Computação, Engenharia de Automação e Sistemas de Informação nas primeiras semanas de aula para fazer um ciclo de palestras introdutórias à Universidade e aos respectivos cursos, palestras de egressos e alunos veteranos sobre perspectivas profissionais, experiências no meio acadêmico e dificuldades encontradas. Também é realizado aulas abordando os fundamentos computacionais, a fim de preencher o vazio deixado pelo ensino médio e assim preparar os alunos para as disciplinas de Introdução à Ciência da Computação, Introdução à Engenharia de Automação, Matemática Discreta e Atividade de Integração Curricular I. O foco principal nessas aulas é construir o pensamento lógico para construção de algoritmos para solução de problemas, livre de qualquer linguagem de programação específica.

Com os resultados obtidos podemos concluir que a primeira edição do projeto teve um grande impacto principalmente no curso de Engenharia de Computação onde houve o maior índice de participação dos estudantes. No Gráfico 4 encontramos as médias de aprovação dos três cursos envolvidos nos últimos três anos.

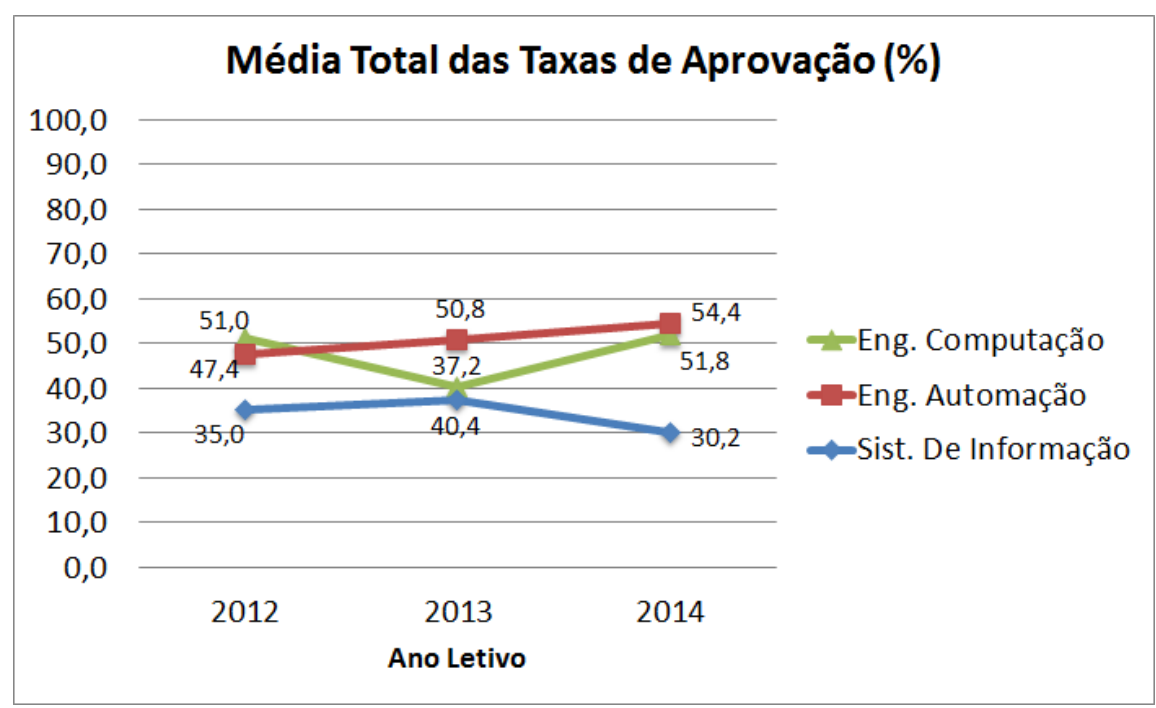

\section{Gráfico 4. Média total das Taxas de Aprovação das disciplinas em cada cursos envolvido no projeto, em porcentagem (\%).}

A segunda edição do projeto ocorreu no início do ano letivo vigente e portanto não podemos obter resultados em relação ao aproveitamento das disciplinas. No entanto, vários alunos que participaram nesta segunda edição já mostrou interesse em participar em vários projetos da Universidade, principalmente no Programa de Educação Tutorial onde todos os inscritos para a seleção que estavam no primeiro ano da graduação haviam participado do Pré-Comp. Esse comportamento reflete diretamente a motivação dos estudantes em relação aos cursos que escolheram e o interesse no desenvolvimento na área da ciência e tecnologia. Outro comportamento interessante observado na segunda edição e que está diretamente relacionado com o nível de motivação dos estudantes foi a participação dos alunos da primeira edição do projeto como tutores.

Como trabalhos futuros, buscamos intensificar a participação dos alunos nas próximas edições do projeto. Para isso aumentaremos as formas de divulgação nas 


\section{CBIE-LACLO 2015}

Anais dos Workshops do IV Congresso Brasileiro de Informática na Educação (CBIE 2015)

turmas, e uma logo já foi elaborada para identidade visual do projeto. E também uma outra métrica para entender os perfis que ingressam na universidade assim como compreender as expectativas que os influenciaram a escolher os cursos que optaram é utilizar questionários de interesse e nível motivacional. A partir da análise dos questionários podemos adequar as atividades do projeto $\mathrm{e}$ contribuir com $\mathrm{o}$ planejamento pedagógico dos cursos.

\section{Referência Bibliográfica}

Andrade, G. M. D. P. C., Fernandes, G. L., de Oliveira, L. B., \& Mendonça, P. C. C. (2013). Revisão bibliográfica sobre o ensino universitário brasileiro: um estudo sobre as novas políticas públicas de acesso e permanência à educação superior. IX ENPEC - Encontro Nacional de Pesquisa em Educação em Ciências.

McWhorter, W. I. and O'Connor, B. C. (2009). "Do LEGO® Mindstorms ${ }^{\circledR}$ motivate students in CS1?", In Proceedings of the 40th ACM technical symposium on Computer science education (SIGCSE '09). ACM, New York, NY, USA, 438-442. DOI=10.1145/1508865.1509019 http://doi.acm.org/10.1145/1508865.1509019

Ministério da Educação Brasileiro. (2007). "O Plano de desenvolvimento da educação: razões, princípios e programas". Brasília: Instituto Nacional de Estudos e Pesquisas Educacionais Anísio Teixeira.

Mitchell, M., Sheard, J., and Markham, S. (2000). "Student motivation and positive impressions of computing subjects". In Proceedings of the Australasian conference on Computing education (ACSE '00), Ainslie E. Ellis (Ed.). ACM, New York, NY, USA, $189-194$. DOI $=10.1145 / 359369.359398$ http://doi.acm.org/10.1145/359369.359398

Silveira, F. L. D., Barbosa, M. C. B., \& Silva, R. D. (2015). Exame Nacional do Ensino Médio (ENEM): Uma análise crítica. Revista Brasileira de Ensino de Física, 37(1), 1101 .

Simon, B., Hanks, B., McCauley, R., Morrison, B., Murphy, L., and Zander, C. (2009). "For me, programming is ...". In Proceedings of the fifth international workshop on Computing education research workshop (ICER '09). ACM, New York, NY, USA, 105-116.

DOI $=10.1145 / 1584322.1584335$ http://doi.acm.org/10.1145/1584322.1584335

Stipek, D.J. (1988). "Motivation to learn: from theory to practice." Englewood Cliffs, New Jersey, Prentice Hall, 1988. 178 páginas.

Tew, A. E., Fowler, C., and Guzdial, M. (2005). "Tracking an innovation in introductory CS education from a research university to a two-year college". SIGCSE Bull. 37, 1 (February 2005), $\quad 416-420$. DOI $=10.1145 / 1047124.1047481$ http://doi.acm.org/10.1145/1047124.1047481

Wick, M. R. (2007). "Bridging the conceptual gap: assessing the impact on student attitudes toward programming". In Proceedings of the 38th SIGCSE technical symposium on Computer science education (SIGCSE '07). ACM, New York, NY, 
CBIE-LACLO 2015

Anais dos Workshops do IV Congresso Brasileiro de Informática na Educação (CBIE 2015)

USA,

$509-513$.

DOI $=10.1145 / 1227310.1227483$

http://doi.acm.org/10.1145/1227310.1227483

Zimpeck, A. L. ; Maciel, B. K ; Soares, L. B. ; Aguiar, Y. Q. ; Oliveira, V. M. ; Amaral, R. S. (2012) "Acolhendo e colaborando com o aperfeiçoamento acadêmico do Centro de Ciências Computacionais". In: $11^{a}$ Mostra da Produção Universitária (MPU). In: $11^{\text {a }}$ Mostra da Produção Universitária (MPU), 2012, Rio Grande. 11ª Mostra da Produção Universitária (MPU). 